\section{CVIA}

REVIEW ARTICLE

pISSN 2508-707X / eISSN 2508-7088 https://doi.org/10.22468/cvia.2017.00178 CVIA 2018;2(2):65-75

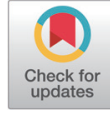

\title{
Assessment of Myocardial Ischemia Using Stress Perfusion Cardiovascular Magnetic Resonance
}

\author{
Akimasa Yamada, Masaki Ishida, \\ Kakuya Kitagawa, Hajime Sakuma \\ Department of Radiology, Mie University Hospital, Tsu, Japan
}

Coronary artery disease (CAD) is a major cause of mortality and morbidity throughout the world, and its prevalence is expected to increase worldwide. Myocardial ischemia is a strong predictor of future adverse outcomes in patients with suspected CAD. The detection of ischemia is an important part of the diagnostic strategy for patients with suspected CAD. Stress myocardial perfusion cardiovascular magnetic resonance (CMR) is a noninvasive diagnostic approach for the detection of myocardial ischemia. CMR offers several advantages over single-photon emission computed tomography, such as high spatial resolution and no radiation exposure. Stress perfusion CMR offers high diagnostic performance for the detection of CAD and prognostic value in patients with suspected CAD. In this review, we describe how to perform stress perfusion CMR and interpret its findings. We also discuss recent updates concerning the diagnostic and prognostic value of stress perfusion CMR in patients with suspected CAD.

Key words Magnetic resonance imaging - Myocardial perfusion imaging · Coronary artery disease $\cdot$ Myocardial ischemia.

Received: December 11, 2017

Revised: March 26, 2018

Accepted: April 3, 2018

Corresponding autho

Masaki Ishida, MD, PhD

Department of Radiology,

Mie University Hospital,

2-174 Edobashi, Tsu,

Mie 514-8507, Japan

Tel: 81-59-231-5029

Fax: 81-59-232-8066

E-mail: mishida@clin.medic.mie-u.ac.jp nostic value has also been documented $[14,15]$.

The purpose of this review is to describe how to obtain and interpret stress perfusion CMR images and to report its diagnostic and prognostic value in patients with suspected CAD.

\section{HOW TO PERFORM STRESS PERFUSION CMR}

\section{Preparation of the magnetic resonance imaging environment}

Performing CMR examinations requires the incorporation of additional physiological monitoring into general magnetic resonance (MR) equipment, such as electrocardiogram (ECG) monitoring, MR-compatible blood pressure monitoring with remote activation from the control room, and oxygen saturation monitoring [16]. Most MR systems currently use advanced triggering modules based on vector-cardiography (VCG). During positioning, the quality of the ECG trace should be checked. If the tracing is unsatisfactory, repositioning of ECG electrodes is required to obtain an optimum signal. An MR-compatible power injector and separate infusion pump are required to deliver an intravenous bolus of contrast agent and continuous infusion

(c) This is an Open Access article distributed under the terms of the Creative Common Attribution Non-Commercial License (http://creativecommons.org/licenses/bync/4.0) which permits unrestricted non-commercial use, distribution, and reproduction in any medium, provided the original work is properly cited. 
of adenosine (Fig. 1). A crash cart including a defibrillator and adequate expertise in basic and advanced cardio-respiratory life support must be available throughout the CMR examination to address any adverse events.

\section{Patient preparation}

Patients are instructed to abstain from substances containing caffeine $12-24 \mathrm{~h}$ prior to stress testing, as caffeine attenuates the coronary hyperemic response to pharmacologic stress agents by blocking the $\mathrm{A}_{2 \mathrm{~A}}$ receptors [17]. Generally, 2 intravenous lines are placed in the antecubital vein in each arm: one for gadolinium and one for a stress agent. A blood pressure cuff is placed on either arm with care not to interfere with the injection of the gadolinium or stress agent. MR-compatible ECG electrodes and the receiver coil are placed on the chest. It is essential to explain the breath-hold commands to the patient and, if necessary, briefly practice these instructions.

\section{Pharmacological stressors}

Stress perfusion CMR is usually performed under pharmacologic stress. Various pharmacologic stress agents, such as ade-

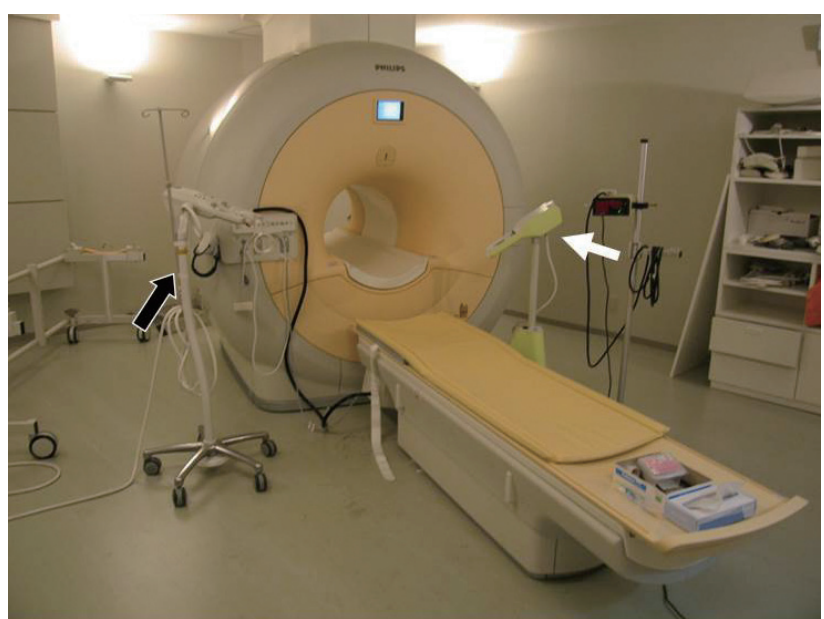

Fig. 1. Typical example of an MRI unit equipped with a power injector with a dual injection system (contrast agent and saline) (black arrow) and an MR-compatible infusion system for the administration of vasodilator agents (white arrow). nosine, dipyridamole and regadenoson, are used to induce vasodilation $[18,19]$. These pharmacologic stress agents relax the arteriolar tonus through the stimulation of $\mathrm{A}_{2 \mathrm{~A}}$ receptors and are associated with a 3- to 5-fold increase in myocardial blood flow (MBF) in normal subjects [20] (Table 1). Adenosine is the most widely used stress agent for myocardial perfusion CMR. Adenosine activates $A_{1}, A_{2 A, B}$, and $A_{3}$ receptors non-selectively and has an extremely short half-life ( $<10$ seconds) [18], allowing the side effects to be resolved quickly by termination of the adenosine infusion. Adenosine is administered at an infusion dose of $140 \mu \mathrm{g} / \mathrm{kg} / \mathrm{min}$ for 3-4 minutes [16]. In patients showing an inadequate hemodynamic response (heart rate increase $<10 \mathrm{bpm}$ or systolic blood pressure decrease $<10 \mathrm{~mm} \mathrm{Hg}$ ) to the standard adenosine protocol, a high-dose adenosine protocol up to $210 \mu \mathrm{g} / \mathrm{min} / \mathrm{kg}$ should be considered [16]. Dipyridamole is an inhibitor of adenosine reuptake that acts indirectly by increasing the local concentration of endogenous adenosine and is activated by metabolism in the liver. The vasodilatory capacity of dipyridamole depends on the individual metabolic rate [18]. Side effects of dipyridamole can be resolved by administration of theophylline [18]. However, a prolonged duration of action for dipyridamole (approximately 30 minutes) makes it less controllable, and it is infrequently used today. Perfusion CMR with dipyridamole uses an infusion dose of $0.56 \mathrm{mg} / \mathrm{kg}$ for 4 minutes, with the peak of vasodilation reached at about 2 minutes after finishing the infusion [21]. Regadenoson (initial half-life 2-4 $\min$ ) is a selective $A_{2 \mathrm{~A}}$ agonist given as an intravenous bolus at a fixed dose of $400 \mu \mathrm{g}$ [19]. Owing to selective $\mathrm{A}_{2 \mathrm{~A}}$ receptor stimulation, regadenoson has a lower incidence of significant side effects and less effect on blood pressure than adenosine [19]. The longer half-life of regadenoson brings the disadvantage of a longer persistence of side effects, although the adverse event rate is lower than that with adenosine or dipyridamole. Regadenoson is administered intravenously 1 minute before contrast injection, reducing the need for infusion pumps and 2 intravenous lines by giving a bolus injection at a fixed dose $[16,19]$. Table 1 summarizes the characteristics of the stress agents.

Adenosine 5'-triophosphate (ATP) is a precursor of endogenous adenosine with a very short half-life $(<10$ seconds) and

Table 1. Characteristics of stress agents

\begin{tabular}{llll}
\hline \multicolumn{1}{c}{ Stress agent } & Dose and infusion rate & \multicolumn{1}{c}{ Mechanism of action } & Half-life \\
\hline Dipyridamole & $0.56 \mathrm{mg} / \mathrm{kg}$ & Inhibiting the adenosine reuptake & $30 \mathrm{~min}$ \\
& $4-\mathrm{min}$ infusion & & $<10 \mathrm{sec}$ \\
Adenosine & $140-210 \mu \mathrm{g} / \mathrm{kg} / \mathrm{min}$ & Activation of $\mathrm{A}_{1}, \mathrm{~A}_{2 \mathrm{~A}, \mathrm{~B}}, \mathrm{~A}_{3}$ receptors & \\
& $3-5-\mathrm{min} \mathrm{infusion}$ & & Same as adenosine \\
Adenosine 5'-triphosphate (ATP) & $160 \mu \mathrm{g} / \mathrm{kg} / \mathrm{min}$ & Same as adenosine & \\
& $3-5-\mathrm{min}$ infusion & & $2-4$ min \\
Regadenoson & $0.4 \mathrm{mg}$ & Activation of $\mathrm{A}_{2 \mathrm{~A}}$ receptor & \\
& Single bolus & & \\
\hline
\end{tabular}


effects similar to adenosine [22]. The main advantage of ATP is the reduced cost [23]. There are also fewer side effects with intravenous infusion ATP than adenosine, an important factor in the clinical setting [24,25]. Accordingly, ATP is widely used as a pharmacologic stress agent instead of adenosine for stress myocardial perfusion imaging, mainly in Asian countries [26-29] and some European countries [23,25]. ATP is administered intravenously at $0.16 \mathrm{mg} / \mathrm{kg} / \mathrm{min}$ [22,24-29]. Watanabe et al. [26] found a similar diagnostic efficacy with dipyridamole $(0.156 \mathrm{mg} /$ $\mathrm{kg} / \mathrm{min})$ and ATP $(0.16 \mathrm{mg} / \mathrm{kg} / \mathrm{min})$. Chun et al. [27] directly compared the effect of adenosine $(0.14 \mathrm{mg} / \mathrm{kg} / \mathrm{min})$ and ATP $(0.16 \mathrm{mg} / \mathrm{kg} / \mathrm{min})$ in the same subjects using Tl-201 SPECT and reported that the hemodynamic changes and degree of myocardial uptake were similar between adenosine and ATP.

Contra-indications for these stress agents include second- or third-degree atrioventricular block, systolic blood pressure $<90$ $\mathrm{mm} \mathrm{Hg}$, sinus bradycardia (heart rate $<40 \mathrm{bpm}$ ), reactive airway disease with regular use of inhalers and known hypersensitivity to these agents $[16,30]$. Side effects for these stress agents may include light flushing, mild chest discomfort and headache. More serious side effects include atrioventricular conduction block, bronchospasm, a rapid decrease in blood pressure or cerebral hypoperfusion with potential neurological symptoms in the presence of significant carotid stenosis [30].

Splenic switch-off is a novel sign in the assessment of stress adequacy during adenosine myocardial stress perfusion CMR [31]. The spleen has an abundant blood flow in the resting state. However, splanchnic vasoconstriction is induced by the administration of adenosine. Therefore, the spleen has reduced signal intensity during stress compared with the resting state on perfusion imaging (Fig. 2). Failed splenic switch-off with adenosine is an observation that identifies understressed patients who are at risk of having false-negative findings on stress perfusion CMR (Fig. 3). Using splenic switch-off as the assessment
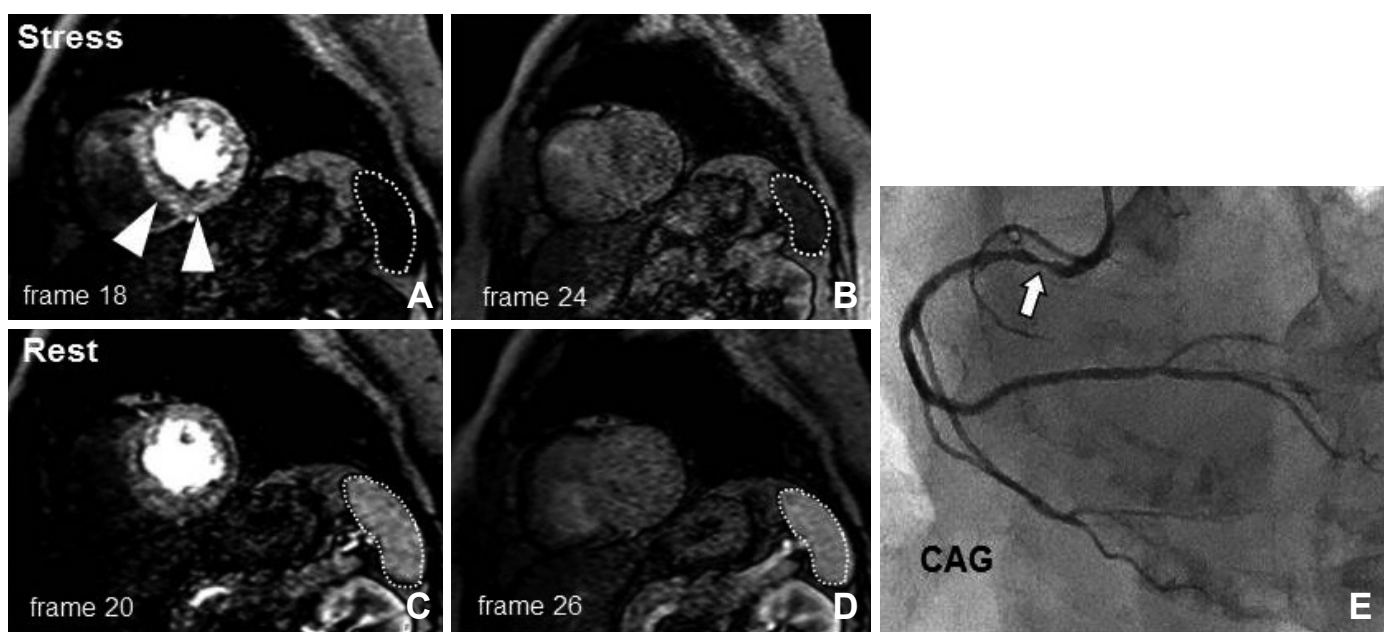

Fig. 2. A case with splenic switch-off. In this 81-year-old woman with chest oppression, the spleen exhibits reduced signal intensity during stress $(A$ and $B)$ compared with the resting state $(C$ and $D)$ on perfusion imaging (dotted circles). CAG reveals significant stenosis in the RCA (white arrow) (E). In this patient, stress-induced ischemia due to significant stenosis in the RCA is accurately detected by stress perfusion cardiovascular magnetic resonance in the inferior wall (arrowheads) (A). CAG: coronary angiography, RCA: right coronary artery.
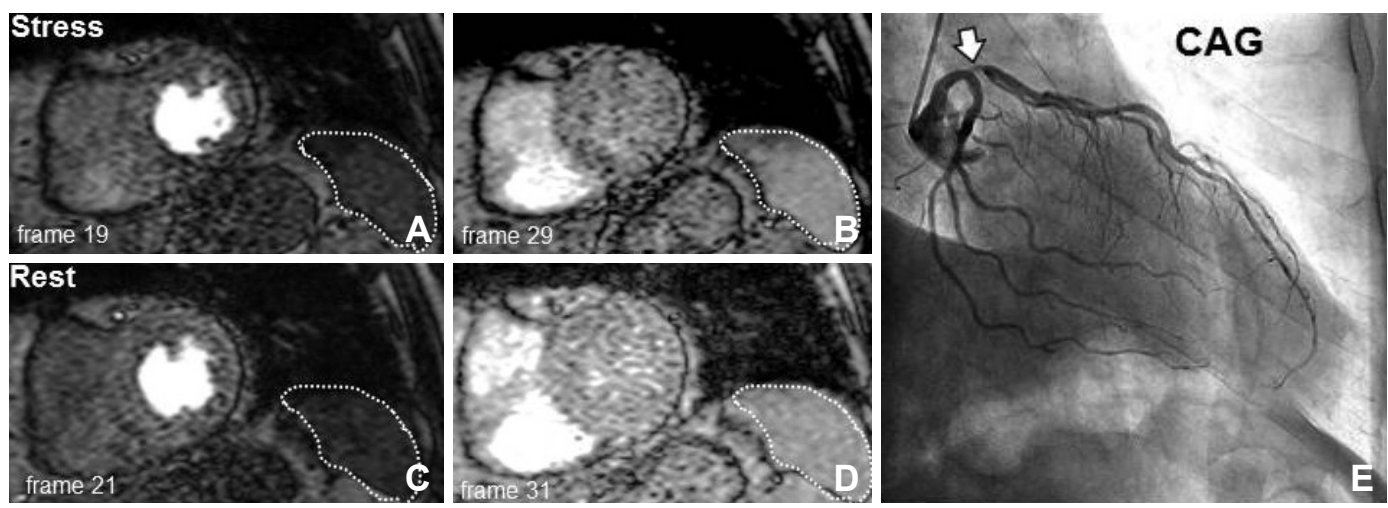

Fig. 3. A case with failed splenic switch-off. In this 73-year-old man with dyspnea on exertion, the spleen signal intensity on perfusion CMR was not reduced during stress ( $A$ and $B$ ) compared with perfusion CMR at rest (C and D) (dotted circles). In this patient, stress perfusion CMR failed to detect stress-induced ischemia despite the presence of significant stenosis in the proximal left anterior descending artery on CAG (arrow) (E). CMR: cardiovascular magnetic resonance, CAG: coronary angiography. 
for adequate pharmacological response may improve the test sensitivity of stress perfusion magnetic resonance imaging (MRI). However, pharmacologic stress perfusion CMR acquired after the administration of regadenoson shows no attenuation of splenic blood flow. Therefore, splenic switch-off as the assessment for adequate pharmacological response cannot apply to stress perfusion imaging with regadenoson.

\section{Perfusion CMR imaging}

An extracellular gadolinium-based contrast agent is used for first-pass myocardial perfusion CMR. It is recommended that CMR be performed according to the standardized CMR protocols proposed by the Society for Cardiovascular Magnetic Resonance [16]. Stress perfusion CMR is generally performed as part of a comprehensive CMR protocol including cine MRI, perfusion MRI, and late gadolinium-enhanced (LGE) imaging. After obtaining a true short axis view of the left ventricle and before starting pharmacological stress, a "test" perfusion sequence without contrast should be acquired to assess the presence of artifacts, satisfactory VCG triggering and patient compliance with breathing commands. The patients are instructed to begin holding their breath at the start of image acquisition and to continue breath-holding for as long as possible followed by shallow breathing in order to minimize respiratory artifacts. It is important to obtain data every heart beat with at least 3 slices per beat during 40-50 RR intervals. Immediately after perfusion CMR acquisition is started, the contrast agent is injected as a bolus into a peripheral vein at a dose of $0.05-0.10$ $\mathrm{mmol} / \mathrm{kg}$ and a rate of 3-7 mL/s, followed by a $30-\mathrm{mL}$ saline flush using the same injection rate to facilitate a compact bolus passage $[3,16]$.

\section{HOW TO INTERPRET STRESS PERFUSION CMR}

Myocardial perfusion CMR can be evaluated by visual, semiquantitative and quantitative analyses [5,32]. Semi-quantitative and quantitative analyses rely on time intensity curves measured from regions of interest in the left-ventricular (LV) blood and myocardium. A widely available post-processing software program is lacking at present for quantitative analysis. Furthermore, performing a quantitative analysis can be difficult because the MR signal from the LV blood pool is no longer proportional to the gadolinium concentration during the first pass [32]. Consequently, semi-quantitative and quantitative analyses of perfusion CMR are largely restricted to research applications. Accordingly, we will focus on the visual assessment of stress perfusion CMR in this review.

After the intravenous bolus administration of contrast agents, there is pronounced signal enhancement in the right-ventricular
(RV) cavity, followed by the LV cavity and, finally, the LV myocardium. When coronary arteries are narrowed by atherosclerotic disease, coronary autoregulation attempts to normalize the MBF by reducing the resistance of distal perfusion beds to preserve adequate myocardial oxygen supply $[33,34]$. Since autoregulation already causes compensatory maximal dilation at rest in the stenosis-dependent myocardium, these vessels cannot be dilated any further. Thus, pharmacologic vasodilation induces an increase in the blood flow in myocardial areas supplied by normal coronary arteries, whereas no changes are found in areas supplied by stenotic coronary arteries $[33,34]$. As a result, normal myocardium will show a homogenous increase in the signal intensity, followed by contrast washout. Conversely, the area supplied by coronary arteries with significant stenosis will show a delayed signal intensity increase. This manifests as a darker area, known as a perfusion defect, in the poorly perfused myocardium (Fig. 4). A true perfusion defect is characterized by being most prominent in the subendocardium, occurring in segments that anatomically correlate to a particular coronary artery distribution and persisting for several RR intervals [35].

Perfusion images are generally assessed using the American Heart Association (AHA) 16-segment model. Rest and stress perfusion images are displayed side by side on a workstation and visually evaluated by manually paging the images. In addition, corresponding slices of LGE images are presented. Table 2 summarizes how to interpret stress perfusion CMR using LGE. First, LGE images are reviewed to obtain information regarding the presence or absence of myocardial infarction (MI), and then the stress and rest perfusion CMR images are evaluated for ischemia [35] (Fig. 5). Ischemia is considered present if a myocardial perfusion defect is present during stress that was not observed at rest in the absence of MI. If a myocardial perfusion defect is seen in both rest and stress perfusion CMR images and there is no evidence of scarring on LGE images, the defect is considered an artifact or resting ischemia.

Several important pitfalls should be acknowledged when interpreting perfusion CMR. The most common and annoying artifact is dark rim artifact (DRA) [36], which typically occurs at the interface between the LV cavity and subendocardial border and can be mistaken for a true perfusion defect. It may mimic a subendocardial perfusion defect in its location but has characteristics that allow it to be differentiated from a true perfusion defect. DRA often appears darker than the non-enhanced myocardium before contrast arrival and thus darker than a true perfusion defect (Fig. 6). It tends to occur before the start of myocardial enhancement and lasts for only a few heart beats before rapidly decreasing in severity and disappearing during the washout phase of contrast. Myocardial hypointensities that do not correspond to an artery distribution territory may be a clue in distinguishing DRA from true perfusion defects [37]. Arti- 

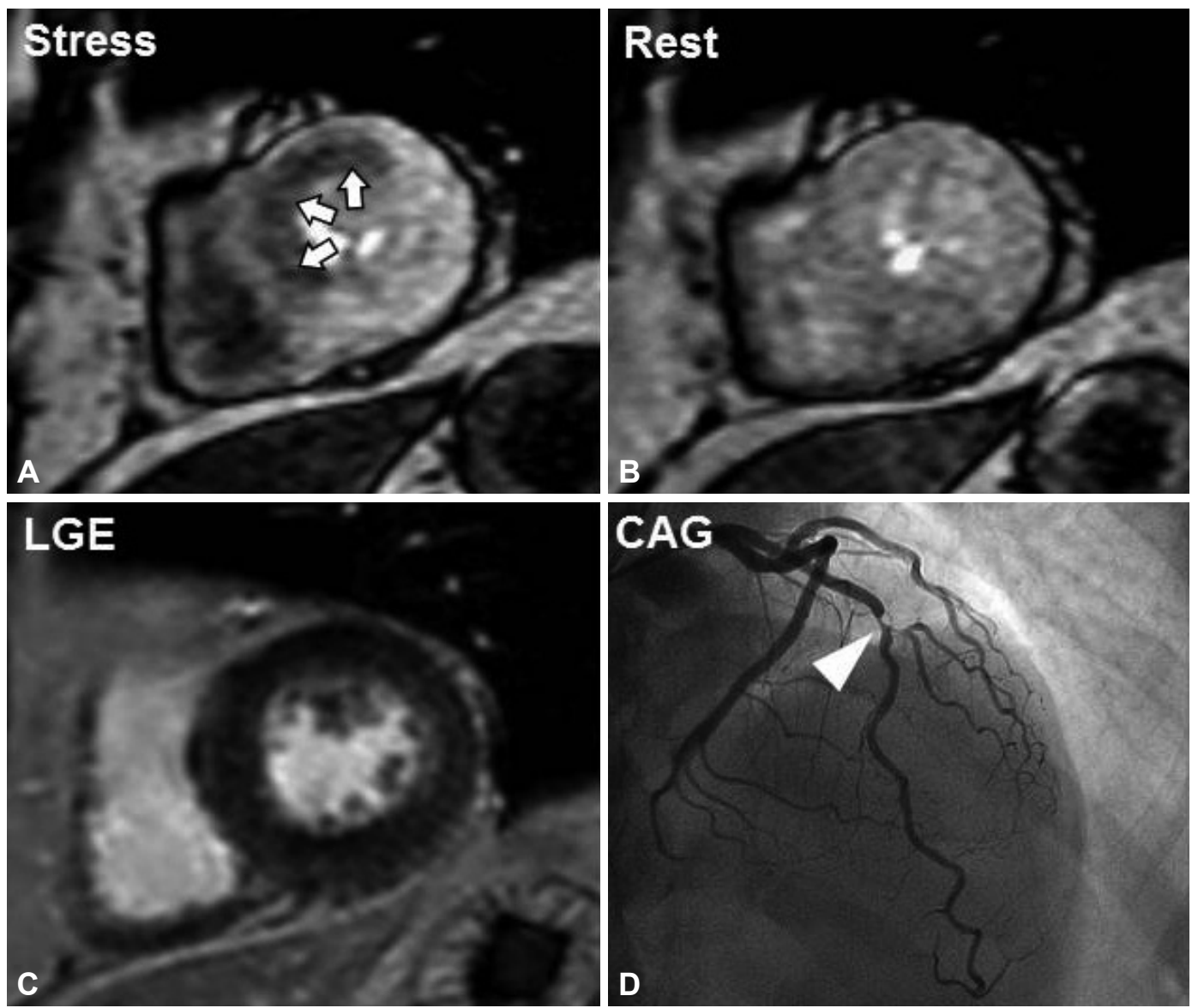

Fig. 4. First-pass myocardial perfusion CMR images during ATP stress $(A)$ and in the resting state $(B)$ in a patient with chest pain. The stress perfusion CMR image shows an area of hypoperfusion in the anteroseptal wall (arrows) that is not depicted in the rest perfusion image, indicating ischemic myocardium due to significant stenosis in the LAD artery. The corresponding LGE image shows the absence of myocardial infarction (C). CAG shows significant coronary artery stenosis (arrowhead) in the LAD artery (D). CMR: cardiovascular magnetic resonance, ATP: adenosine 5'-triophosphate, LAD: left anterior descending, LGE, late gadolinium-enhanced MRI, CAG: coronary angiography.

Table 2. Visual analysis of cardiac perfusion images

\begin{tabular}{llll}
\hline \multicolumn{1}{c}{ Stress } & \multicolumn{1}{c}{ Rest } & \multicolumn{1}{c}{ LGE } & \multicolumn{1}{c}{ Interpretation } \\
\hline Negative & Negative & Negative & Normal \\
Negative & Negative & Positive & Infarction \\
Positive & Negative & Negative & Ischemia \\
Positive & Positive & Positive & Infarction \\
Positive & Positive & Negative & $\begin{array}{c}\text { Artifacts, resting ischemia, } \\
\text { delayed contrast arrival }\end{array}$ \\
& & &
\end{tabular}

LGE: late gadolinium-enhanced MRI

fact mechanisms that may lead to DRA include Gibbs artifact caused by limited resolution, susceptibility artifacts from the passage of the contrast and cardiac motion during data acquisition [36]. The prominence of such artifacts can be reduced by increasing spatial resolution in the phase-encoding direction, as this minimizes Gibbs artifact, which is thought to play an important role in the appearance of DRA [36].

Both multi-vessel disease and microvascular dysfunction have been reported to show a similar entire subendocardial perfusion defect during stress perfusion CMR [38]. With a visual analysis of the images, it is sometimes difficult to distinguish between multi-vessel disease and microvascular dysfunction. The addition of coronary morphology imaging, such as whole-heart coronary MR angiography or coronary CT angiography, to perfusion CMR can help distinguish between multi-vessel disease and microvascular dysfunction (Fig. 7).

Coronary tortuosity and ectasia are phenomena often encountered and caused by age or arteriosclerosis [39]. Contrast agents passing through a tortuous coronary artery must travel a longer distance to the myocardium than when passing through normal coronaries. Tortuosity and ectasia of the coronary artery may lead to delayed regional contrast arrival, resulting in an increased number of false positive results (Fig. 8). The delay caused by the longer distance of the bolus to reach the myocardium should be transmural, while with a true perfusion defect, hypoenhancement is non-transmural in the majority of cases. 
Myocardial Ischemia Assessment by Stress Perfusion CMR

\section{DIAGNOSTIC PERFORMANCE OF STRESS PERFUSION CMR}

\section{Stress perfusion CMR vs. SPECT}

SPECT, commonly used worldwide for the assessment of myocardial ischemia, may have suboptimal diagnostic accuracy in patients with multi-vessel CAD because of equilibration of ischemia [40]. Stress perfusion CMR imaging yields high diagnostic accuracy for the detection of CAD, especially at the subendocardial level, due to its inherent high spatial resolution [35]. Many comparative studies with SPECT suggest that stress perfusion CMR can realistically be used as an alternative modality with superior performance relative to that of SPECT. In our single-center studies that directly compared stress perfusion
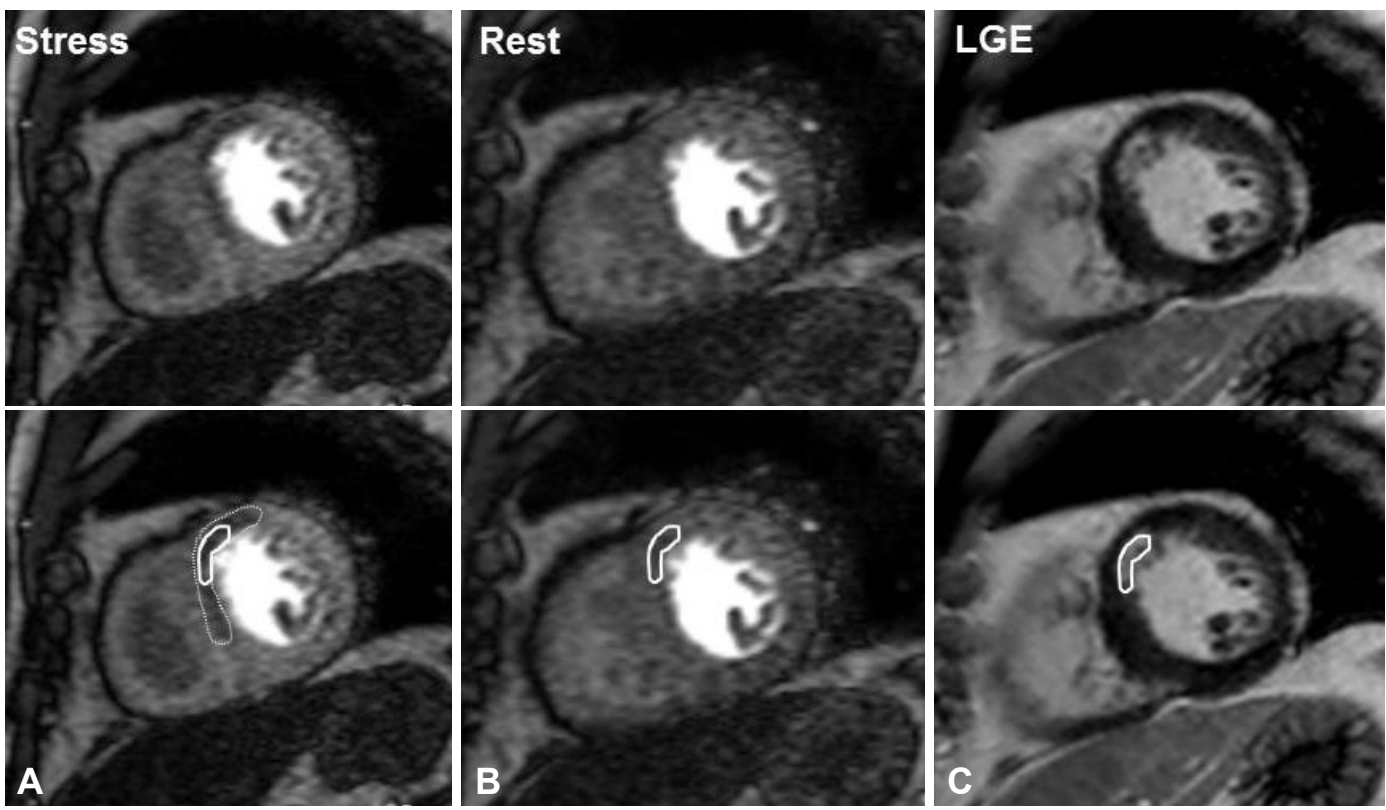

Fig. 5. Interpretation of stress perfusion CMR in relation to LGE CMR. When interpreting a stress CMR study, myocardial perfusion CMR images during stress $(A)$ and at rest $(B)$ and corresponding LGE CMR images (C) are displayed side-by-side. The top and bottom rows represent the same images with and without markers. In the LGE images (C), subendocardial MI is noted in the anteroseptal wall (white circle). The stress and rest images are evaluated for ischemia in the area outside of the Ml region, indicated by the white circle. A myocardial perfusion defect is present during stress that was not observed at rest in the absence of Ml (dotted circle), indicating stress-induced myocardial ischemia. CMR: cardiovascular magnetic resonance, LGE: late gadolinium-enhanced MRI, MI: myocardial infarction.
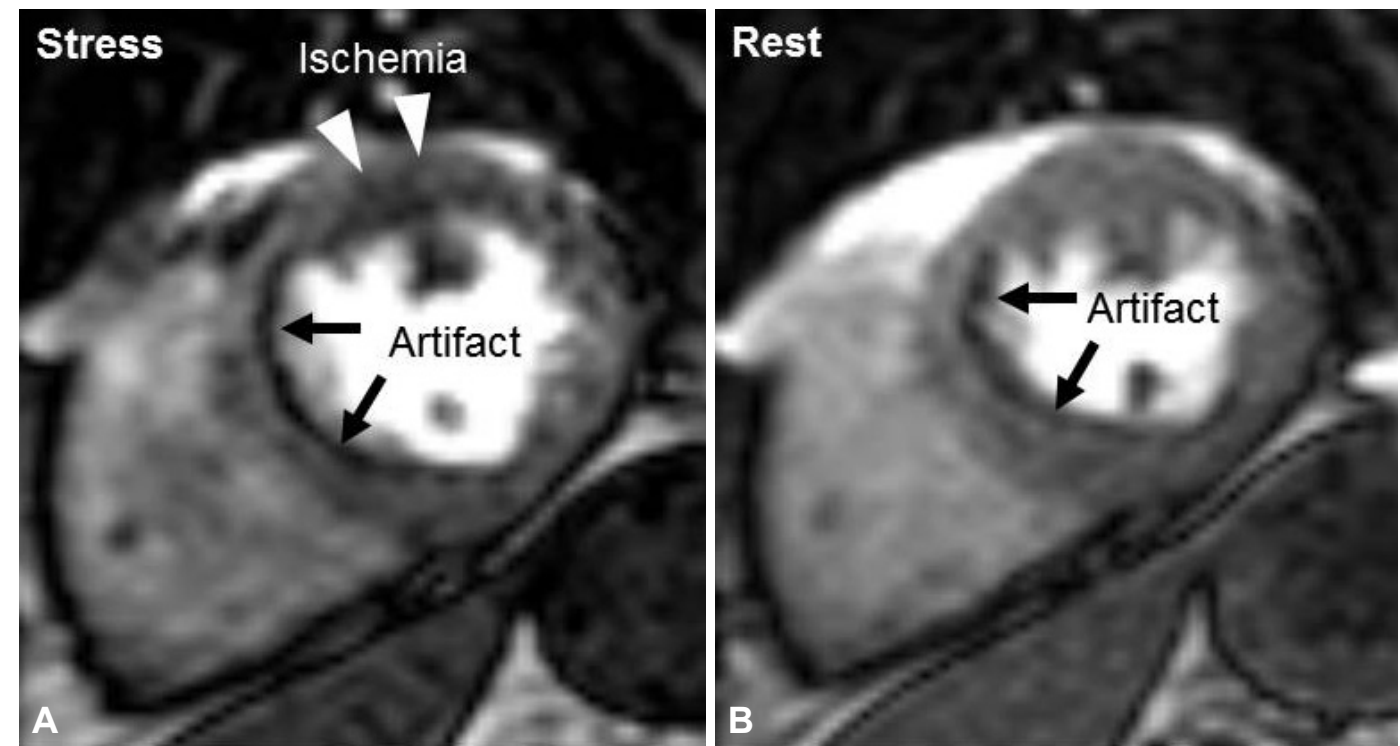

Fig. 6. DRA. ATP stress (A) and rest (B) perfusion CMR images show a DRA at the interface between the left ventricular cavity and subendocardial border of the septum (black arrows). True ischemia can be seen in the anterior wall of the stress perfusion CMR image (white arrowheads) in this case. DRA: dark rim artifact, ATP: adenosine 5'-triophosphate, CMR: cardiovascular magnetic resonance. 

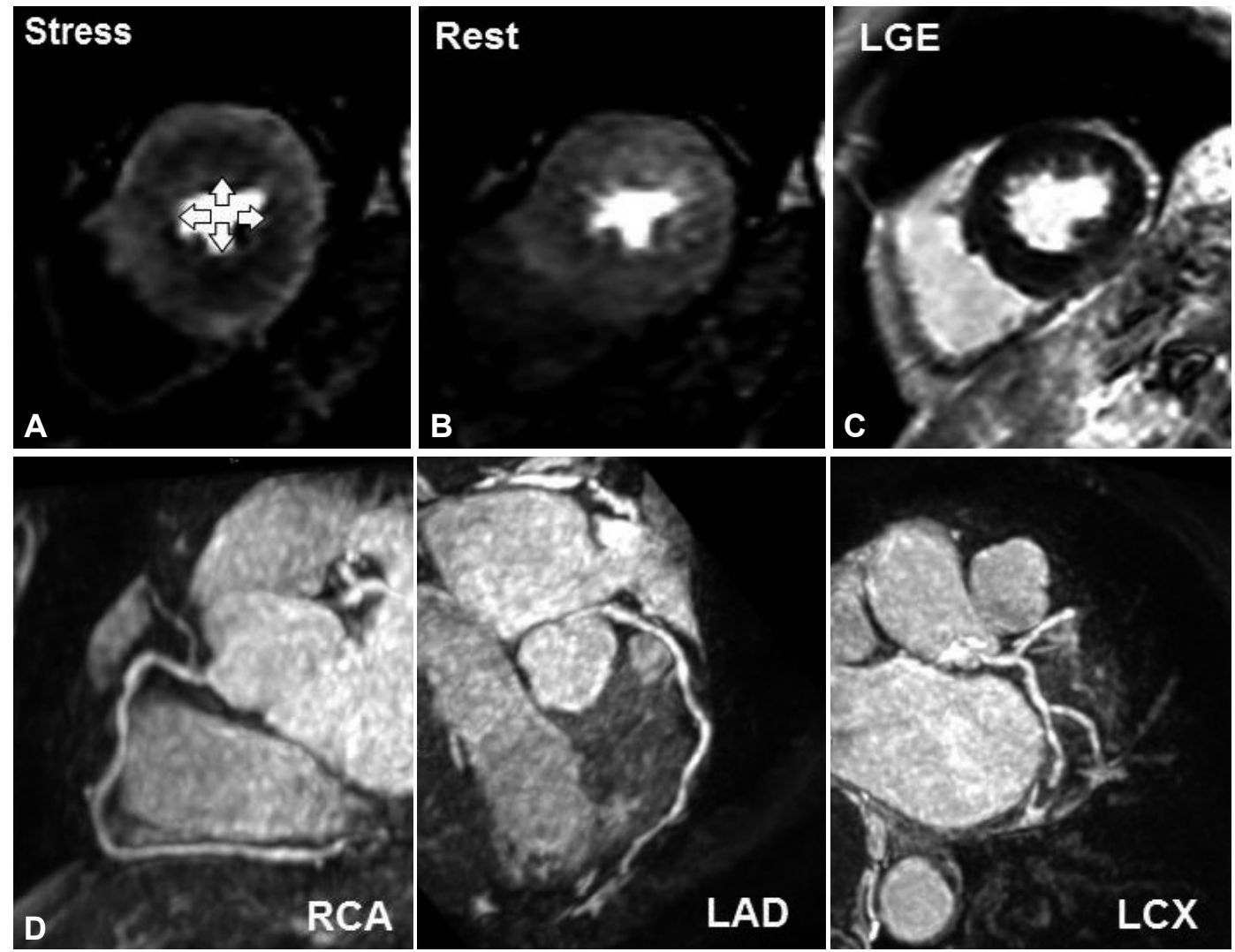

Fig. 7. A 64-year-old woman with chest pain and hypertension. Subendocardial hypoperfusion is observed in the entire circumference of the left ventricle on stress perfusion magnetic resonance imaging (arrows) (A and B) and no myocardial infarction was evident on LGE image $(C)$, suggesting that this patient might have multi-vessel disease or microvascular dysfunction. Multi-vessel disease was excluded by coronary magnetic resonance angiography (D). Note that this patient has a thickened left-ventricular wall. Subendocardial hypoperfusion is frequently observed in patients with left-ventricular hypertrophy, including those with hypertrophic cardiomyopathy and a hypertensive heart. LGE: late gadolinium-enhanced MRI, RCA: right coronary artery, LAD: left anterior descending artery, LCX: left circumflex artery.

CMR and SPECT, the area under the receiver operating characteristic curve (AUC) for the detection of significant CAD [defined as $>50 \%$ luminal narrowing on quantitative coronary angiography (QCA)] was 0.91 for perfusion CMR, which was significantly higher than that for SPECT $(0.75, \mathrm{p}<0.001)$ in a vessel-based analysis [5]. In the previous multicenter, multivendor prospective MR-IMPACT trial, the diagnostic performance of stress perfusion CMR (AUC $0.86 \pm 0.06$ ) was better than that of stress perfusion SPECT $(0.67 \pm 0.05, \mathrm{p}=0.013)$, particularly in patients with 2- and 3-vessel disease [3]. Recently, the CE-MARC study, a prospective study comparing stress CMR and SPECT in a large real-world population, demonstrated that stress perfusion CMR (AUC 0.89) significantly out-performed stress SPECT (AUC 0.74, $\mathrm{p}<0.0001$ ) with higher sensitivity (CMR, 87\%; SPECT, 67\%) and higher negative predictive values (CMR, 91\%; SPECT, 79\%) than SPECT in a patient-based analysis [4]. The CE-MARC study also revealed that stress perfusion CMR performed better than SPECT not only in patients with multi-vessel CAD (AUC: CMR 0.91, SPECT 0.77, p<0.0001) but also in those with single-vessel CAD (AUC: CMR 0.87, SPECT 0.71, p <0.0001) (Table 3) [4]. In a previous meta-analy- sis comparing the diagnostic accuracy of perfusion CMR, SPECT, and positron emission tomography (PET) using QCA as the reference standard, the patient-based analysis per imaging modality demonstrated pooled sensitivities of 88,89 , and $84 \%$ and specificities of 61,76 , and $81 \%$ for SPECT, CMR, and PET, respectively. CMR showed a significantly higher diagnostic accuracy than SPECT [6].

\section{Diagnostic accuracy of stress perfusion CMR against QCA and FFR}

QCA is a well-established technique for the anatomic assessment of CAD [41]. Multiple studies have assessed the ability of perfusion CMR to detect myocardial ischemia with QCA or a visual estimation of stenosis severity as a reference standard, and the high diagnostic accuracy of stress perfusion CMR has been proven [7-9]. A meta-analysis by Nandalur et al. [9] showed that stress perfusion CMR provides a sensitivity of 0.91 and a specificity of 0.81 on a patient level (disease prevalence $=57.4 \%$ ) when compared with QCA as a reference standard.

However, anatomical narrowing of the coronary artery does not always correlate with the functional significance of CAD. 
Although QCA reveals anatomical narrowing of the coronary artery, it may not reliably detect whether or not stenosis leads to ischemia. At present, the fractional flow reserve (FFR), a meth- od of assessing hyperemic pressure differences across coronary artery stenosis, is considered the gold standard for the diagnosis of ischemia-causing CAD [42]. Watkins et al. [10] compared
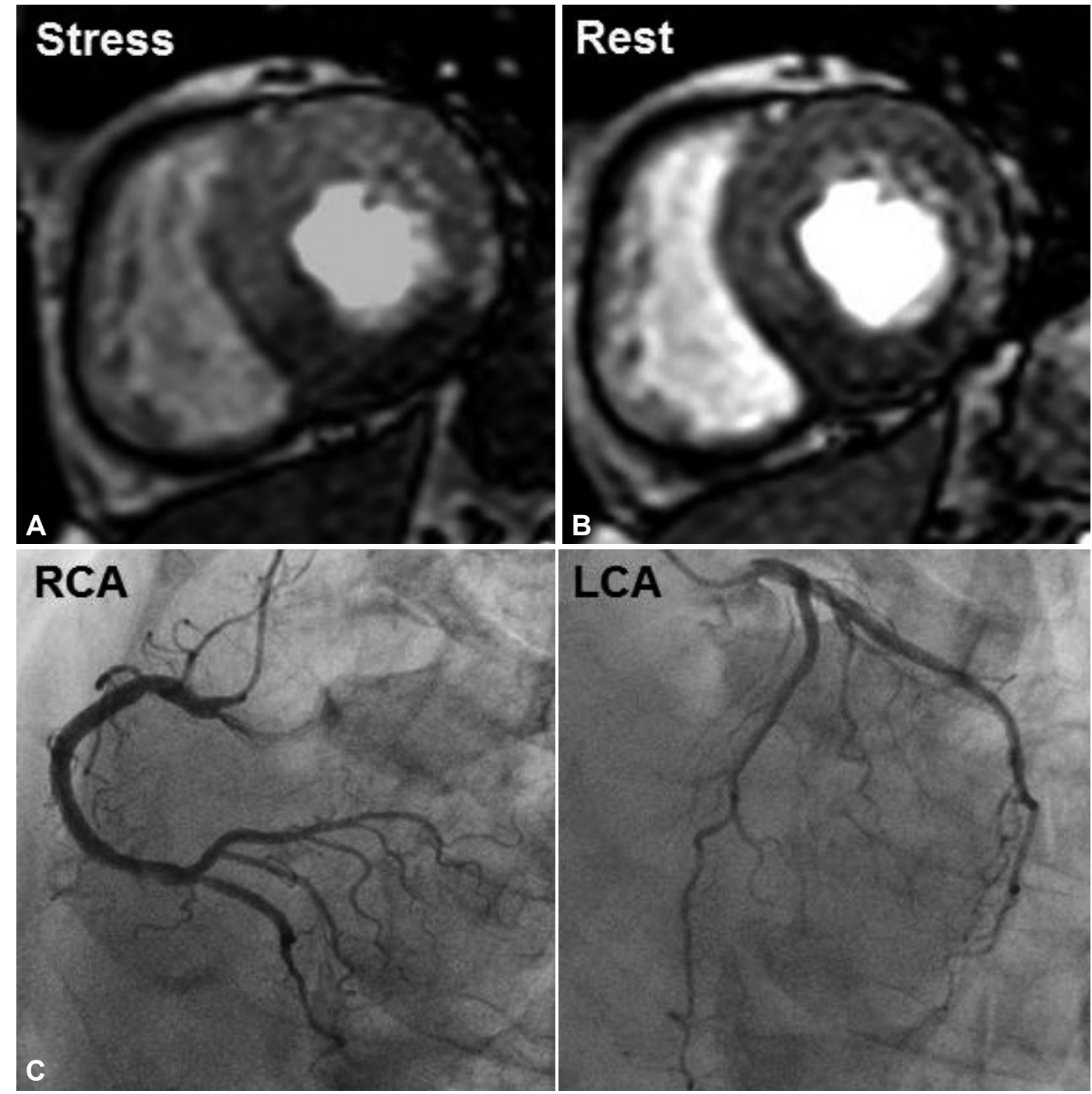

Fig. 8. A transmural perfusion defect without subendocardial predominance is seen on both stress (A) and rest (B) perfusion CMR without evidence of myocardial infarction on late gadolinium-enhanced CMR (not shown). The X-ray coronary angiogram shows normal coronary arteries without atherosclerotic changes $(\mathrm{C})$. Contrast agent passing through a tortuous RCA must travel a longer distance to the myocardium than through a normal LCA, which may lead to delayed regional contrast arrival. CMR: cardiovascular magnetic resonance, RCA: right coronary artery, LCA: left coronary artery.

Table 3. Stress perfusion CMR vs. SPECT

\begin{tabular}{|c|c|c|c|c|c|}
\hline \multirow{2}{*}{$\begin{array}{l}\text { First author } \\
\text { (reference no.) }\end{array}$} & \multirow{2}{*}{ Study } & \multirow{2}{*}{$\begin{array}{l}\text { All/single vessel disease/ } \\
\text { multi-vessel disease }\end{array}$} & \multicolumn{2}{|c|}{ AUC } & \multirow{2}{*}{$\mathrm{p}$ value } \\
\hline & & & CMR & SPECT & \\
\hline Ishida et al. [5] & Single-center $(\mathrm{n}=69)$ & All* & 0.91 & 0.75 & $<0.001$ \\
\hline \multirow[t]{2}{*}{ Schwitter et al. [3] } & Multi-center $(\mathrm{n}=241)$ & All* & 0.86 & 0.67 & 0.013 \\
\hline & & Multi-vessel disease* & 0.89 & 0.70 & 0.006 \\
\hline \multirow[t]{3}{*}{ Greenwood et al. [4] } & Single-center, randomized $(\mathrm{n}=676)$ & $\mathrm{All}^{+}$ & 0.89 & 0.74 & $<0.0001$ \\
\hline & & Single-vessel disease ${ }^{\dagger}$ & 0.87 & 0.71 & $<0.0001$ \\
\hline & & Multi-vessel disease $^{\dagger}$ & 0.91 & 0.77 & $<0.0001$ \\
\hline
\end{tabular}

${ }^{*}>50 \%$ diameter stenosis on QCA, ${ }^{\dagger}>70 \%$ ( $>50 \%$ left main coronary trunk) diameter stenosis on QCA. CMR: cardiovascular magnetic resonance, SPECT: single-photon emission computed tomography, AUC: area under the receiver operating characteristic curve, QCA: quantitative coronary angiography 
Table 4. Diagnostic accuracy of stress perfusion CMR against QCA and FFR

\begin{tabular}{|c|c|c|c|c|c|}
\hline First author (reference no) & Study & Sensitivity (\%) & Specificity (\%) & AUC & Reference \\
\hline Ishida et al. [5] & Single-center $(n=69)$ & 90 & 85 & 0.91 & QCA \\
\hline Greenwood et al. [4] & Single-center $(\mathrm{n}=676)$ & 86.5 & 83.4 & 0.89 & QCA \\
\hline Watkins et al. [10] & Single-center $(\mathrm{n}=103)$ & 95 & 91 & NA & FFR \\
\hline Lockie et al. [11] & Single-center $(n=42)$ & 82 & 94 & 0.92 & FFR \\
\hline \multirow[t]{2}{*}{ Jaarsma et al. [6] } & Meta-analysis studies $(\mathrm{n}=27)$ & 89 & 76 & 0.90 & QCA \\
\hline & Patients $(\mathrm{n}=2841)$ & & & & \\
\hline \multirow[t]{2}{*}{ Nandalur et al. [9] } & Meta-analysis studies $(\mathrm{n}=14)$ & 91 & 81 & NA & QCA \\
\hline & Patients $(\mathrm{n}=1183)$ & & & & \\
\hline \multirow[t]{2}{*}{ Li et al. [12] } & Meta-analysis studies $(\mathrm{n}=14)$ & 90 & 87 & 0.95 & FFR \\
\hline & Patients $(\mathrm{n}=650)$ & & & & \\
\hline \multirow[t]{2}{*}{ Takx et al. [13] } & Meta-analysis studies $(\mathrm{n}=15)$ & 89 & 87 & 0.94 & FFR \\
\hline & Patients ( $\mathrm{n}=798)$ & & & & \\
\hline
\end{tabular}

CMR: cardiovascular magnetic resonance, QCA: quantitative coronary angiography, FFR: fractional flow reserve, AUC: area under the receiver operating characteristic curve

the diagnostic accuracy of perfusion CMR versus FFR (a value $<0.75$ denoting significant stenosis). In a per-patient analysis, the sensitivity and specificity of perfusion CMR for the detection of functionally significant CAD were $95 \%$ and $91 \%$, respectively, with respective positive and negative predictive values of $97 \%$ and $84 \%$ [10]. Lockie et al. [11] also compared perfusion CMR against FFR (a value $<0.75$ denoting significant stenosis). The sensitivity and specificity of visual CMR analysis to detect significant stenosis by FFR were 0.82 and 0.94 , respectively, with an AUC of 0.92 [11]. Thus, stress perfusion CMR can detect functionally significant CAD with excellent diagnostic accuracy. In a meta-analysis reported by Li et al. [12], perfusion CMR allowed for the accurate detection of ischemic $\mathrm{CAD}$ with FFR as the reference standard. The pooled sensitivity, specificity, and AUC were $0.90,0.87$, and 0.95 at the patient level and $0.89,0.86$, and 0.93 at the vessel level, respectively. Another recent meta-analysis comparing stress perfusion CMR with other myocardial perfusion imaging modalities such as PET, SPECT, perfusion computed tomography and perfusion echocardiography, using FFR as a reference, concluded that stress perfusion CMR (sensitivity $89 \%$, specificity $87 \%$ ) has better diagnostic performance than SPECT (sensitivity $74 \%$, specificity $79 \%$ ) or echocardiography (sensitivity $69 \%$, specificity $84 \%$ ) and achieves similar high diagnostic performance compared with perfusion PET (sensitivity $84 \%$, specificity $87 \%$ ) and perfusion computed tomography (sensitivity $88 \%$, specificity $80 \%$ ) (Table 4 ) [13].

\section{PROGNOSTIC VALUE OF PERFUSION CMR}

Over the past several years, multiple studies have demonstrated that stress perfusion CMR has excellent prognostic characteristics and may help guide risk stratification of patients with known or suspected CAD $[14,15]$. According to a recent meta-analysis of 19 intermediate-term follow-up studies using stress CMR, including 15 studies with vasodilator stress perfusion CMR, patients with ischemia had a higher incidence of nonfatal MI [odds ratio (OR): 5.4], cardiovascular death (OR: 5.9) and the combined endpoint (OR: 6.5) than those with a negative stress perfusion CMR study [14]. In addition, patients without evidence of ischemia on stress perfusion CMR have an annual event rate of $<1 \%$ for either cardiovascular death or nonfatal MI, whereas patients with ischemia on stress perfusion CMR have a 5\% annual event rate [14].

SPECT has excellent prognostic value for risk stratification and guiding therapeutic decision-making in patients with known or suspected CAD [43]. Recently, the prognostic value of CMR was compared with that of SPECT in patients with suspected CAD in a 5-year follow-up of the CE-MARC study [15]. The data indicated that CMR is a stronger predictor of risk for major adverse cardiac events (MACEs) than SPECT, independent of cardiovascular risk factors, angiography results, or initial patient treatment, although abnormal findings on CMR (hazard ratio, 2.77; $\mathrm{p}<0.001$ ) and SPECT (hazard ratio, 1.62; $\mathrm{p}=0.014$ ) were both strong and independent predictors of MACEs [15].

\section{SUMMARY}

With adequate imaging and interpretation skills, stress myocardial perfusion CMR provides an accurate assessment of myocardial ischemia and allows for risk stratification and prognostic evaluation in patients with known or suspected CAD.

\section{Conflicts of Interest}

The authors declare that they have no conflict of interest. 


\section{REFERENCES}

1. Thom T, Haase N, Rosamond W, Howard VJ, Rumsfeld J, Manolio T, et al. Heart disease and stroke statistics--2006 update: a report from the American Heart Association Statistics Committee and Stroke Statistics Subcommittee. Circulation 2006;113:e85-e151.

2. Hachamovitch R, Berman DS, Shaw LJ, Kiat H, Cohen I, Cabico JA, et al. Incremental prognostic value of myocardial perfusion single photon emission computed tomography for the prediction of cardiac death: differential stratification for risk of cardiac death and myocardial infarction. Circulation 1998;97:535-543.

3. Schwitter J, Wacker CM, van Rossum AC, Lombardi M, Al-Saadi N, Ahlstrom $\mathrm{H}$, et al. MR-IMPACT: comparison of perfusion-cardiac magnetic resonance with single-photon emission computed tomography for the detection of coronary artery disease in a multicentre, multivendor, randomized trial. Eur Heart J 2008;29:480-489.

4. Greenwood JP, Maredia N, Younger JF, Brown JM, Nixon J, Everett CC, et al. Cardiovascular magnetic resonance and single-photon emission computed tomography for diagnosis of coronary heart disease (CE-MARC): a prospective trial. Lancet 2012;379:453-460.

5. Ishida N, Sakuma H, Motoyasu M, Okinaka T, Isaka N, Nakano T, et al. Noninfarcted myocardium: correlation between dynamic first-pass contrast-enhanced myocardial MR imaging and quantitative coronary angiography. Radiology 2003;229:209-216.

6. Jaarsma C, Leiner T, Bekkers SC, Crijns HJ, Wildberger JE, Nagel E, et al. Diagnostic performance of noninvasive myocardial perfusion imaging using single-photon emission computed tomography, cardiac magnetic resonance, and positron emission tomography imaging for the detection of obstructive coronary artery disease: a meta-analysis. J Am Coll Cardiol 2012;59:1719-1728.

7. Hamon M, Fau G, Née G, Ehtisham J, Morello R, Hamon M. Meta-analysis of the diagnostic performance of stress perfusion cardiovascular magnetic resonance for detection of coronary artery disease. J Cardiovasc Magn Reson 2010;12:29.

8. de Jong MC, Genders TS, van Geuns RJ, Moelker A, Hunink MG. Diagnostic performance of stress myocardial perfusion imaging for coronary artery disease: a systematic review and meta-analysis. Eur Radiol 2012;22: 1881-1895.

9. Nandalur KR, Dwamena BA, Choudhri AF, Nandalur MR, Carlos RC. Diagnostic performance of stress cardiac magnetic resonance imaging in the detection of coronary artery disease: a meta-analysis. J Am Coll Cardiol 2007;50:1343-1353.

10. Watkins S, McGeoch R, Lyne J, Steedman T, Good R, McLaughlin MJ, et al. Validation of magnetic resonance myocardial perfusion imaging with fractional flow reserve for the detection of significant coronary heart disease. Circulation 2009;120:2207-2213.

11. Lockie T, Ishida M, Perera D, Chiribiri A, De Silva K, Kozerke S, et al. High-resolution magnetic resonance myocardial perfusion imaging at 3.0-Tesla to detect hemodynamically significant coronary stenoses as determined by fractional flow reserve. J Am Coll Cardiol 2011;57:70-75.

12. Li M, Zhou T, Yang LF, Peng ZH, Ding J, Sun G. Diagnostic accuracy of myocardial magnetic resonance perfusion to diagnose ischemic stenosis with fractional flow reserve as reference: systematic review and metaanalysis. JACC Cardiovasc Imaging 2014;7:1098-1105.

13. Takx RA, Blomberg BA, El Aidi H, Habets J, de Jong PA, Nagel E, et al. Diagnostic accuracy of stress myocardial perfusion imaging compared to invasive coronary angiography with fractional flow reserve meta-analysis. Circ Cardiovasc Imaging 2015;8:e002666.

14. Lipinski MJ, McVey CM, Berger JS, Kramer CM, Salerno M. Prognostic value of stress cardiac magnetic resonance imaging in patients with known or suspected coronary artery disease: a systematic review and meta-analysis. J Am Coll Cardiol 2013;62:826-838.

15. Greenwood JP, Herzog BA, Brown JM, Everett CC, Nixon J, Bijsterveld P, et al. Prognostic value of cardiovascular magnetic resonance and singlephoton emission computed tomography in suspected coronary heart dis- ease: long-term follow-up of a prospective, diagnostic accuracy cohort study. Ann Intern Med 2016;165:1-9.

16. Kramer CM, Barkhausen J, Flamm SD, Kim RJ, Nagel E; Society for Cardiovascular Magnetic Resonance Board of Trustees Task Force on Standardized Protocols. Standardized cardiovascular magnetic resonance (CMR) protocols 2013 update. J Cardiovasc Magn Reson 2013;15:91.

17. Zoghbi GJ, Htay T, Aqel R, Blackmon L, Heo J, Iskandrian AE. Effect of caffeine on ischemia detection by adenosine single-photon emission computed tomography perfusion imaging. J Am Coll Cardiol 2006;47:22962302.

18. Iskandrian AS, Verani MS, Heo J. Pharmacologic stress testing: mechanism of action, hemodynamic responses, and results in detection of coronary artery disease. J Nucl Cardiol 1994;1:94-111.

19. Hendel RC, Bateman TM, Cerqueira MD, Iskandrian AE, Leppo JA, Blackburn B, et al. Initial clinical experience with regadenoson, a novel selective A2A agonist for pharmacologic stress single-photon emission computed tomography myocardial perfusion imaging. J Am Coll Cardiol 2005;46:2069-2075.

20. Leppo JA. Comparison of pharmacologic stress agents. J Nucl Cardiol 1996;3(6 Pt 2):S22-S26.

21. Albro PC, Gould KL, Westcott RJ, Hamilton GW, Ritchie JL, Williams DL. Noninvasive assessment of coronary stenoses by myocardial imaging during pharmacologic coronary vasodilatation. III. Clinical trial. Am J Cardiol 1978;42:751-760.

22. Belhassen B, Pelleg A. Electrophysiologic effects of adenosine triphosphate and adenosine on the mammalian heart: clinical and experimental aspects. J Am Coll Cardiol 1984;4:414-424.

23. Bravo N, Giménez M, Mejía S, García-Velloso MJ, Coma-Canella I. Prognostic value of myocardial perfusion imaging with adenosine triphosphate. J Nucl Cardiol 2002;9:395-401.

24. Cerqueira MD, Verani MS, Schwaiger M, Heo J, Iskandrian AS. Safety profile of adenosine stress perfusion imaging: results from the Adenoscan Multicenter Trial Registry. J Am Coll Cardiol 1994;23:384-389.

25. Coma-Canella I, Palazuelos J, Bravo N, García Velloso MJ. Myocardial perfusion imaging with adenosine triphosphate predicts the rate of cardiovascular events. J Nucl Cardiol 2006;13:316-323.

26. Watanabe K, Sekiya M, Ikeda S, Miyagawa M, Kinoshita M, Kumano S. Comparison of adenosine triphosphate and dipyridamole in diagnosis by thallium-201 myocardial scintigraphy. J Nucl Med 1997;38:577-581.

27. Chun KA, Lee J, Lee SW, Ahn BC, Ha JH, Cho IH, et al. Direct comparison of adenosine and adenosine 5 '-triphosphate as pharmacologic stress agents in conjunction with Tl-201 SPECT: Hemodynamic response, myocardial tracer uptake, and size of perfusion defects in the same subjects. J Nucl Cardiol 2006;13:621-628.

28. Yao Z, Zhu H, Li W, Chen C, Wang H, Shi L, et al. Adenosine triphosphate stress myocardial perfusion imaging for risk stratification of patients aged 70 years and older with suspected coronary artery disease. J Nucl Cardiol 2017;24:429-433.

29. Tomiyama Y, Manabe O, Oyama-Manabe N, Naya M, Sugimori H, Hirata $\mathrm{K}$, et al. Quantification of myocardial blood flow with dynamic perfusion 3.0 Tesla MRI: validation with (15) O-water PET. J Magn Reson Imaging 2015;42:754-762.

30. Henzlova MJ, Duvall WL, Einstein AJ, Travin MI, Verberne HJ. ASNC imaging guidelines for SPECT nuclear cardiology procedures: stress, protocols, and tracers. J Nucl Cardiol 2016;23:606-639.

31. Manisty C, Ripley DP, Herrey AS, Captur G, Wong TC, Petersen SE, et al. Splenic switch-off: a tool to assess stress adequacy in adenosine perfusion cardiac MR imaging. Radiology 2015;276:732-740.

32. Ichihara $T$, Ishida $M$, Kitagawa $K$, Ichikawa $Y$, Natsume T, Yamaki N, et al. Quantitative analysis of first-pass contrast-enhanced myocardial perfusion MRI using a Patlak plot method and blood saturation correction. Magn Reson Med 2009;62:373-383.

33. Johnson PC. Autoregulation of blood flow. Circ Res 1986;59:483-495.

34. Harrison DG, Florentine MS, Brooks LA, Cooper SM, Marcus ML. The effect of hypertension and left ventricular hypertrophy on the lower range 
of coronary autoregulation. Circulation 1988;77:1108-1115.

35. Schulz-Menger J, Bluemke DA, Bremerich J, Flamm SD, Fogel MA, Friedrich MG, et al. Standardized image interpretation and post processing in cardiovascular magnetic resonance: Society for Cardiovascular Magnetic Resonance (SCMR) board of trustees task force on standardized post processing. J Cardiovasc Magn Reson 2013;15:35.

36. Di Bella EV, Parker DL, Sinusas AJ. On the dark rim artifact in dynamic contrast-enhanced MRI myocardial perfusion studies. Magn Reson Med 2005;54:1295-1299.

37. Cerqueira MD, Weissman NJ, Dilsizian V, Jacobs AK, Kaul S, Laskey WK, et al. Standardized myocardial segmentation and nomenclature for tomographic imaging of the heart. A statement for healthcare professionals from the Cardiac Imaging Committee of the Council on Clinical Cardiology of the American Heart Association. Circulation 2002;105:539-542.

38. Panting JR, Gatehouse PD, Yang GZ, Grothues F, Firmin DN, Collins P, et al. Abnormal subendocardial perfusion in cardiac syndrome $\mathrm{X}$ detected by cardiovascular magnetic resonance imaging. N Engl J Med 2002;346:
1948-1953.

39. Zegers ES, Meursing BT, Zegers EB, Oude Ophuis AJ. Coronary tortuosity: a long and winding road. Neth Heart J 2007;15:191-195.

40. Christian TF, Miller TD, Bailey KR, Gibbons RJ. Noninvasive identification of severe coronary artery disease using exercise tomographic thallium-201 imaging. Am J Cardiol 1992;70:14-20.

41. Bartúnek J, Sys SU, Heyndrickx GR, Pijls NH, De Bruyne B. Quantitative coronary angiography in predicting functional significance of stenoses in an unselected patient cohort. J Am Coll Cardiol 1995;26:328-334.

42. Tonino PA, De Bruyne B, Pijls NH, Siebert U, Ikeno F, van’t Veer M, et al. Fractional flow reserve versus angiography for guiding percutaneous coronary intervention. N Engl J Med 2009;360:213-224.

43. Hachamovitch R, Hayes SW, Friedman JD, Cohen I, Berman DS. Comparison of the short-term survival benefit associated with revascularization compared with medical therapy in patients with no prior coronary artery disease undergoing stress myocardial perfusion single photon emission computed tomography. Circulation 2003;107:2900-2907. 